\title{
PLANEJAMENTO DE ATIVIDADES ANTI-TUBERCULOSE PELO MÉTODO CENDES/OPS
}

\begin{abstract}
ARantes, G. R. - Planejamento de atividades anti-tuberculose pelo método $C E N D E S / O P S$. Rev. Saúde públ., S. Paulo, 10:17-29, 1976.

RESUMO: O método CENDES/OPS foi aplicado no municipio de Araraquara (S. Paulo). O dano tuberculose foi programado de acordo com o modelo normativo. Foram elaborados outros programas com dados simulados, com o objetivo de se verificar a influencia de variações na proporção de vacináveis, incidência da doença e eficácia da vacina BCG. As atividades antituberculose foram programadas segundo as caracteristicas epidemiologicas da área programática. A discrepância entre os resultados foi muito grande, salientando-se que as previsóes orçamentárias efetuadas de acordo com o modelo normativo foram oito vezes superiores ao orçamento elaborado com os dados epidemiológicos da área.
\end{abstract}

Unitermos: Planejamento de Saride. Método CENDES/OPS. Técnica de programação local. Modelo normativo. Área programática. Caracteristicas epidemiológicas.

\section{$1-$ INTRODUCAO}

O processo de planejamento de saúde conhecido como Método CENDES/OPS ${ }^{1}$ ou Técnica de Programação Local tem sido aplicado no Brasil, revelando-se muito bom para o diagnóstico de situaçōes, porém pouco prático para a programação propriamente dita ? Uma das dificuldades reside na insuficiência de normas técnicas ou na existência de normas nem sempre aplicáveis às situaçôes encontradas. A tuberculose em todas as suas formas, classificada como dano** n. ${ }^{\circ} 11$, compreende os itens 010-019 e 511 da classificação internacional de doenças, $8 .^{a}$ revisão ${ }^{6}$. Segundo o modelo de normalização ${ }^{4}$, a técnica para combater esse dano compõe-se de consulta inicial para o diagnóstico, hospitalização de $30 \%$ dos pacientes diagnosticados e uma visita domiciliar inicial; o valor relativo desses instrumentos que compóem a técnica é expresso pelo fator de ponderação 0.86 atribuído à hospitalização e 0,14 atribuído à visitação.

Quanto às concentrações de cada atividade executada pelos instrumentos, a norma preconiza 12 consultas de ambulatório,

\footnotetext{
* Da 6.a Divisão Regional de Saúde (Ribeirão Preto) da Coordenadoria de Saúde da Comunidade. Secretaria da Saúde do Estado de São Paulo - Ribeirão Preto, SP - Brasil.

** Doenças de mesma etiologia ou grupos de doenças de etiologias diferentes, vulneráveis às mesmas açōes de saúcle.
} 
ARANTES, G. R. - Planejamento de atividades anti-tuberculose pelo método CENDES/OPS. Rev. Saúde públ., S. Paulo, 10:17-29, 1976.

90 dias de internação na fase inicial do tratamento e 3 visitas domiciliares, sendo os fatores de ponderação respectivamente $0,67,0,27$ e 0,06 . A imunização com dose única refere-se ao BCG usado via intradérmica ${ }^{7}$.

0 rendimento ótimo dos instrumentos, estabelecido por critério técnico e econômico ${ }^{1}$, prevê 20 imunizações, uma visita e 4 consultas por hora-instrumento respectivo e um rendimento do leito-dia da ordem de $95 \%$. Finalmente a cobertura ideal seria de no mínimo $90 \%$, considerando-se que $48,16 \%$ da populaçāo é suscetível e $34,17 \%$ é vacinável; a probabilidade de adoecer é igual a 2 por 1.000 na população como um todo, sem distinção entre infectados e não infectados. Conseqüentemente, usando-se vacina com $80 \%$ de eficácia ${ }^{5}$, haveria uma probabilidade de adoecer entre os vacinados $80 \%$ menor, ou seja, de 0,4 por 1.000 .

Embora as normas referentes à composição da técnica, concentrações e rendimentos sejam discutíveis, sua aplicação não traz maiores dificuldades em nosso meio.

Alguns aspectos referentes às normas de cobertura são bastante criticáveis. Embora não haja contra-indicação em vacinar pessoas já infectadas, somente os não infectados são considerados vacináveis; ora, o número destes depende da prevalência de doentes bacilíferos e das condições de transmissibilidade da doença, sendo de se esperar, portanto, grandes variações de uma área para outra. Logo, não seria adequado o uso de uma mesma norma para todas as áreas.

A eficácia da vacina BCG segundo estudos controlados realizados em diferentes regiões do globo ${ }^{10}$, revelou-se variável de 14 até $82 \%$, possivelmente devido à elevada prevalência de infecçóes por micobactérias atípicas nas regiôes onde a eficácia foi pequena. Presume-se que nas regiões brasileiras mais próximas do equador possa existir esse tipo de interferência e por isso esse fator também deveria ser levado em consideração. A incidência da moléstia não é a mesma nas diversas regiōes brasileiras e por isso as probabilidades de adoecer não são as mesmas em todo o país. Além do mais, a incidência da doença entre infectados é diferente da incidência entre não infectados, embora isso não seja levado em conta pelo método.

0 presente trabalho, baseado em dados reais e situações hipotéticas, pretende demonstrar a necessidade de se levar em conta esses fatores para que o planejamento anti-tuberculose pelo método em questão possa ser melhor efetuado em nosso meio.

\section{2 - MATERIAL E METODOS}

A técnica de programação local foi aplicada no município de Araraquara, Estado de São Paulo, pelos alunos do V Curso de Especialização em Planejamento do Setor Saúde, ministrado pela Faculdade de Saúde Pública da USP em 1972. Estendendo-se por $1.541 \mathrm{~km}^{2}$, sua população total era de 104.742 habitantes em 1971, dos quais cem mil foram considerados como accessíveis.

Os dados necessários foram obtidos, segundo um roteiro pré-estabelecido, nas agências oficiais existentes no município, no Ambulatório da Cooperativa dos Fornecedores de Cana, na Santa Casa de Misericórdia (apenas a morbidade) e no SESA (Serviço Especial de Saúde de Araraquara), sendo esta a unidade sanitária do município de Araraquara, mantido pela Faculdade de Saúde Pública e operando em convênio com a Secretaria da Saúde como um CS-I da rede estadual $^{8}$.

Essa unidade sanitária era a única responsável pela prestação do serviço antituberculose no município. Para isso contava com um clínico geral treinado em Tisiologia, realizava teste tuberculínico padronizado ${ }^{3}$, abreugrafia, radiologia, laboratório, enfermagem, visitação domiciliar e educação sanitária; a vacinação BCG (via oral) era feita em escala diminuta apenas quando solicitada. 
ARANTES, G. R. - Planejamento de atividades anti-tuberculose pelo método CENDES/OPS. Rev. Saúde puibl., S. Paulo, 10:17-29, 1976.

Para obter a produção por danos foram estudadas todas as fichas clínicas do ambulatório e uma amostra de $10 \%$ dos prontuários de pessoas atendidas no SESA, no ano de 1971, selecionados por amostragem sistemática. A técnica preconiza a coleta de dados de produção para os diversos danos, separada em dois itens: total de atendimentos (volume) e atendimentos em primeira consulta. Dividindo-se o volume pelo número de pessoas atendidas em primeira consulta obtém-se a concentração, isto é, o número de vezes que cada paciente foi atendido. No caso em pauta os registros e relatórios não discriminavam o volume referente apenas aos doentes novos. Por essa razão o volume total foi dividido pelo total de doentes (novos no ano $\mathrm{e}$ antigos) para $o$ cálculo da concentração; esta foi então multiplicada pelo número de doentes novos no ano para a obtenção do volume total de atendimentos a esses pacientes.

O método também não prevê gastos com os demais doentes em tratamento, vindos dos anos anteriores, os quais representaram aproximadamente seis vezes o número de casos diagnosticados no ano de 1971. Por isso, a fim de calcular os gastos totais com o atendimento a todos os doentes (tratamento e visitação) os custos com novos doentes foram multiplicados por seis.

As características epidemiológicas da área foram obtidas nos relatórios da seção de epidemiologia e estatística do SESA; como os mesmos se revelassem muito semelhantes aos encontrados em Ribeirão Preto, que é uma área próxima de Araraquara e comparável a ela, usou-se a proporção de vacináveis $(60 \%)$ obtida nessa área *, pois que os dados de Araraquara eram insuficientes para tal. $O$ coeficiente de incidência observado $(0,25$ por 1.000$)$ refere-se a toda a população, infectada ou não. Admitindo-se que ele seja a média ponderada entre o coeficiente (mais baixo) nos $60 \%$ não infectados e o

\footnotetext{
* Dados não publicados.
}

coeficiente (mais alto) nos $40 \%$ infectados, estes seriam, respectivamente, 0,20 e 0,30 por 1.000 .

Com o objetivo de verificar a influência de variações em alguns parâmetros propostos no modelo normativo (proporção de vacináveis, incidência da doença e eficácia da vacina) realizaram-se os seguintes estudos orçamentários com programas alternativos mínimo (apenas atendimentos aos doentes) e máximo (atendimentos e vacinação) para cada um:

Estudo n. 1 : segundo o modelo normativo.

Estudos n. ${ }^{\text {os }} 2$ e 3: segundo variações hipıtéticas na proporção de vacináveis.

Estudo $n .^{\circ} 4$ : segundo variação hipotética na incidência.

Estudos $n{ }^{\text {os }} 5$ e 6 : segundo variações hipotéticas na eficácia da vacina.

Estudos n. ${ }^{\circ}$ 7, 8 e 9: segundo as características epidemiológicas da área e diferentes eficácias da vacina.

Para facilitar o confronto entre esses estudos os resultados foram submetidos a uma análise custo-benefício ${ }^{\circ}$; considerouse como "benefício" a economia em gastos com doentes (alternativa mínima menos alternativa máxima) e "custo" o gasto com imunizações (Tabela 4).

\section{3 - RESULTADOS}

A incidência da moléstia, expressa pelos casos diagnosticados no ano em residentes no município, e a prevalência, pelo total de casos em tratamento, são apresentados na Tabela 1.

Como se pode notar o número de casos novos tem sido em média igual a 1/6 dos doentes em tratamento. A prevalência de infecção, medida pelo índice tuberculínico, revelou os valores apresentados na Tabela 2. 
ARANTES, G. R. - Planejamento de atividades anti-tuberculose pelo método CENDES/OPS. Rev. Saúde públ., S. Paulo, 10:17-29, 1976.

TABEA 1

Incidência e prevalência de tuberculose pulmonar em Araraquara - 1968 a 1971

\begin{tabular}{|c|c|c|c|c|c|}
\hline \multirow[b]{2}{*}{ Ano } & \multicolumn{2}{|c|}{$\begin{array}{c}\text { Casos novos no } \\
\text { ano }\end{array}$} & \multicolumn{2}{|c|}{$\begin{array}{c}\text { Total de casos em } \\
\text { tratamento }\end{array}$} & \multirow{2}{*}{$\begin{array}{c}\text { População } \\
\text { do } \\
\text { municiplo }\end{array}$} \\
\hline & $\begin{array}{l}\text { N.os } \\
\text { absolutos }\end{array}$ & $\begin{array}{l}\text { Por } 1.000 \\
\text { habitantes }\end{array}$ & $\begin{array}{c}\text { N.os } \\
\text { absolutos }\end{array}$ & $\begin{array}{l}\text { Por } 100.000 \\
\text { habitantes }\end{array}$ & \\
\hline 1968 & 24 & 0.25 & 156 & 164 & 94.884 \\
\hline 1969 & 29 & 0.29 & 157 & 162 & 96.678 \\
\hline 1970 & 22 & 0,21 & 150 & 147 & 101.381 \\
\hline 1971 & 27 & 0,25 & 177 & 169 & 104.700 \\
\hline
\end{tabular}

Fonte: Relatórios anuais do Serviço Especial de Saúde de Araraquara (SESA).

TA B E L A 2

Prevalência de reatores fortes ao teste tuberculínico padronizado com 2 U.T. de P.P.D. rt 23 em Araraquara, por grupo etário, de 1969 a 1971.

\begin{tabular}{|c|c|c|c|}
\hline \multirow{2}{*}{ Idade } & \multicolumn{3}{|c|}{ Ano } \\
\hline & 1969 & 1970 & 1971 \\
\hline Menores de 1 ano & 2,750 & $1,2 \%$ & $2,3 \%$ \\
\hline 1 a 4 anos & $2,5 \%$ & 1,95 & $1,8 \%$ \\
\hline 5 a 14 anos & $3,6 \%$ & $4,6 \%$ & $2,9 \%$ \\
\hline 0 a 14 anos & $3,4 \%$ & $4,1 \%$ & $2,7 \%$ \\
\hline 15 a 19 anos & $6,2 \%$ & $8,0 \%$ & $3,9 \%$ \\
\hline 20 a 29 anos * & $12,8 \%$ & $15,1 \%$ & $13,9 \%$ \\
\hline 0 a 29 anos & $5,5 \%$ & $7,1 \%$ & $4,0 \%$ \\
\hline
\end{tabular}

* Acima dessa idade as pessoas são abreugrafadas sem teste tuberculínico prévio.

Fonte: Relatórios Anuais do SESA.

A aplicação dos sucessivos passos do método culminou com a obtenção dos atributos de atividades e instrumentos apresentados na Tabela 3 .

A Tabela 4 contém os resultados dos estudos realizados, inclusive da análise custo-benefício.

0 resumo final do processamento efetuado para cada estudo pode ser visto nas Tabelas 5 a 13.

$$
4 \text { - DISCUSS } \mathrm{AO}
$$

A análise dos resultados apresentados na Tabela 4 revela-nos que a variação na proporção de vacináveis (Estudos $n^{0} 2 \mathrm{e}$ n. ${ }^{\circ}$ 3) provoca importantes alterações nos valores orçamentários em comparação com os valores obtidos segundo as normas preconizadas pelo método (Estudo $n .^{\circ} 1$ ): se apenas $10 \%$ da população fosse vaci- 
ARANTES, G. R. - Planejamento de atividades anti-tuberculose pelo método CENDES/OPS. Rev. Saúde públ., S. Paulo, 10:17-29, 1976.

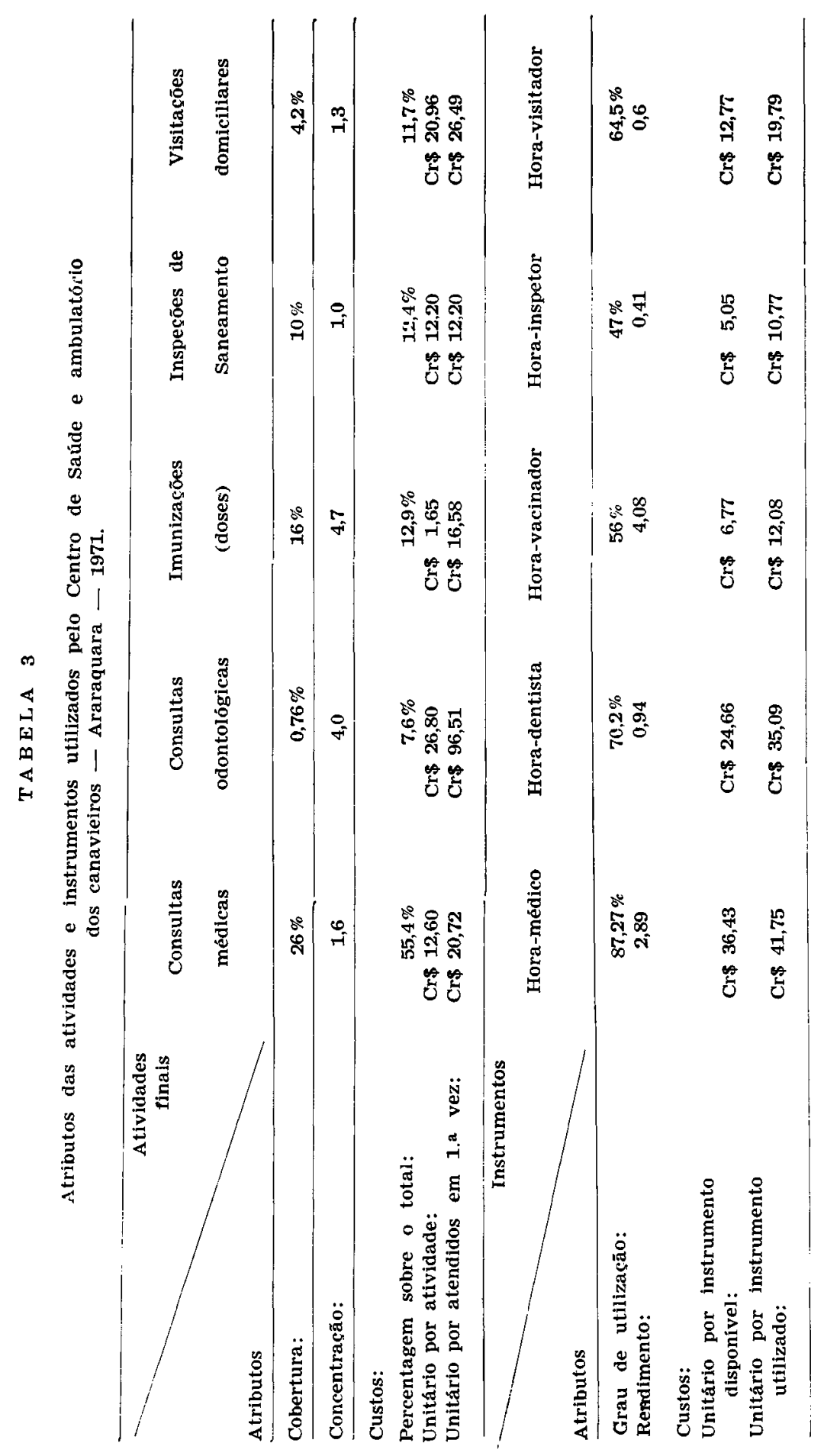


ARANTES, G. R. - Planejamento de atividades anti-tuberculose pelo método CENDES/OPS. Rev. Saúde publ., S. Paulo, 10:17-29, 1976.

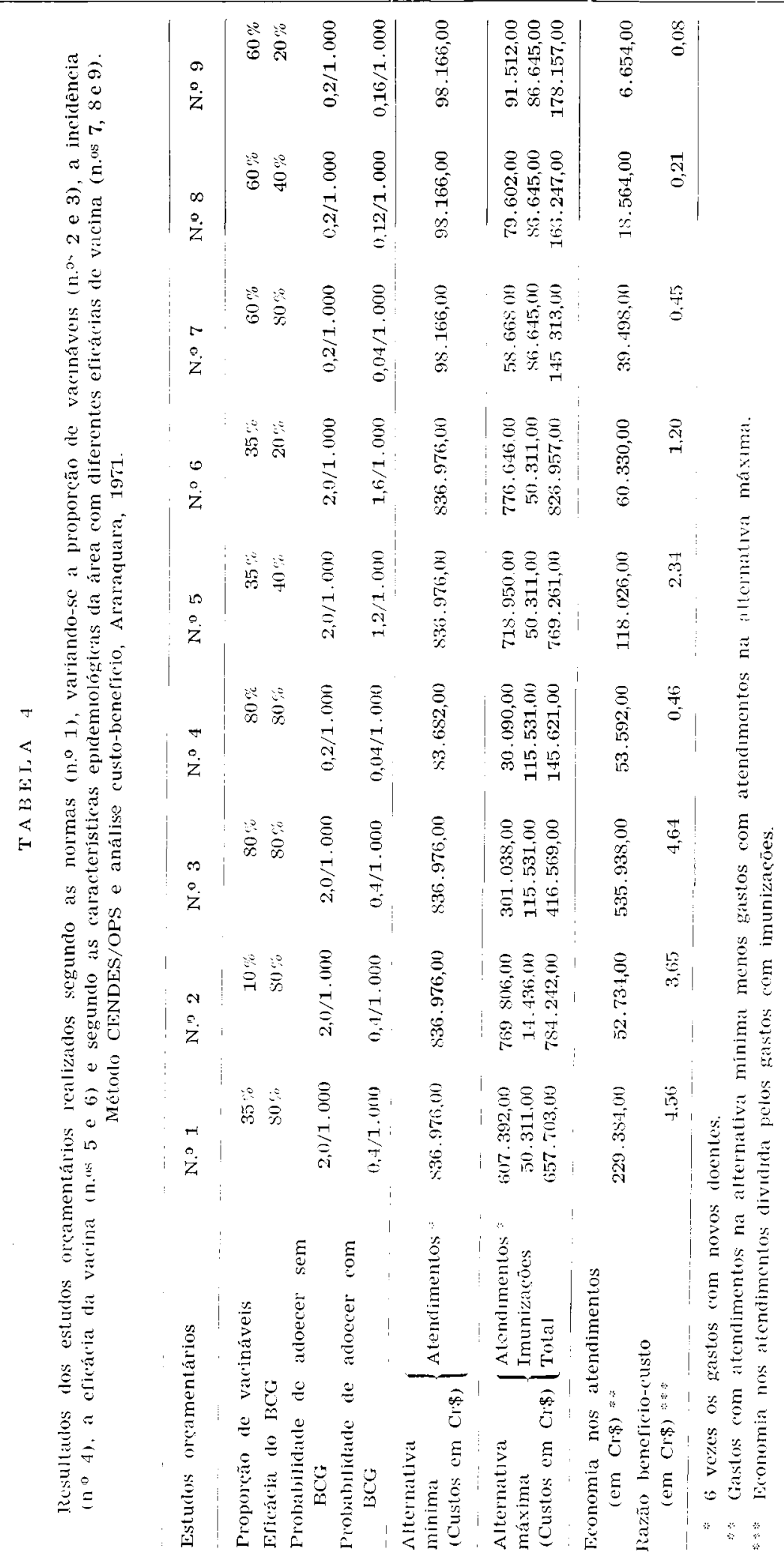


ARANTES, G. R. - Planejamento de atividades anti-tuberculose pelo método CENDES/OPS. Rev. Saúde públ., S. Paulo, 10:17-29, 1976.

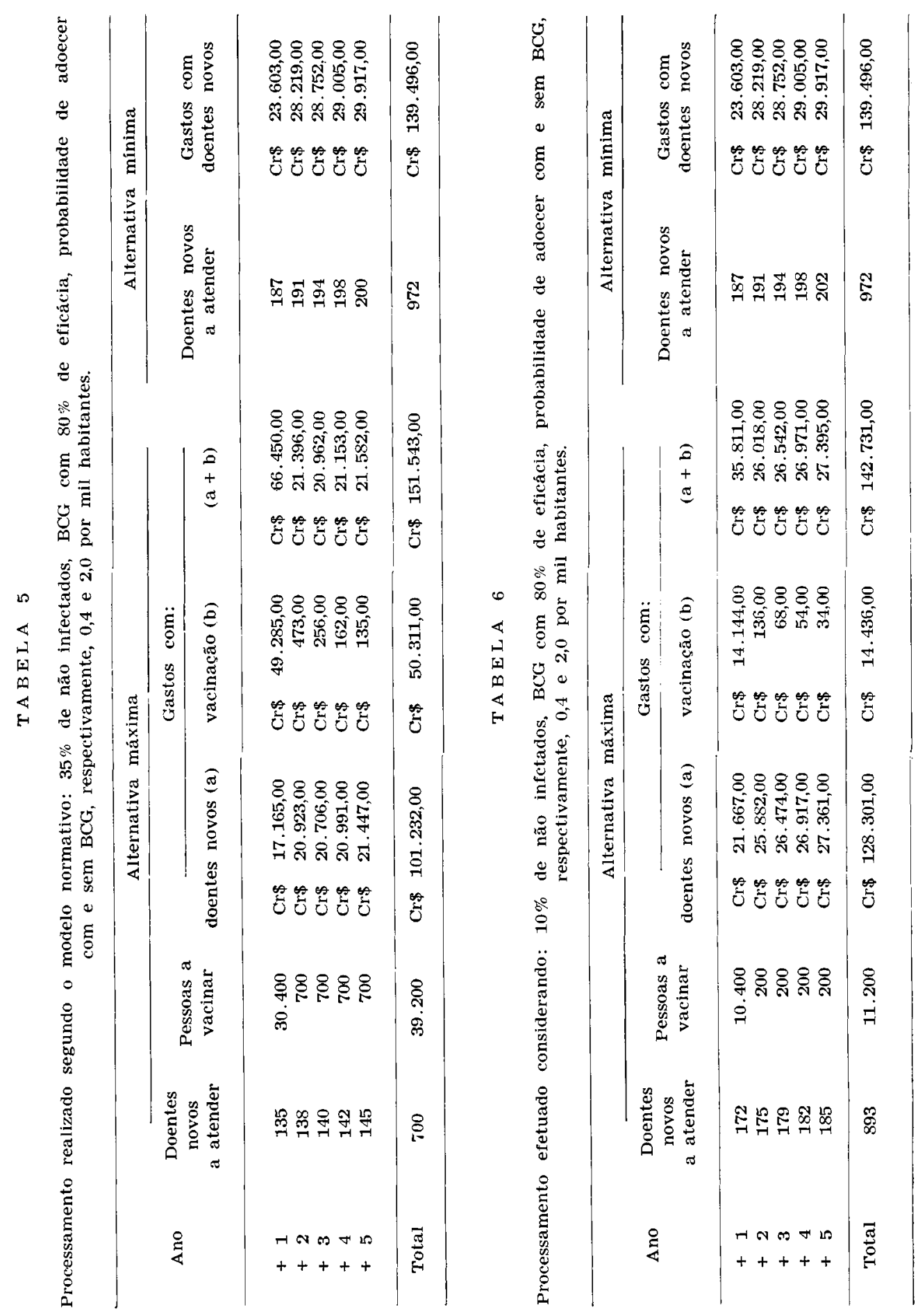


ARANTES, G. R. - Planejamento de atividades anti-tuberculose pelo método CENDES/OPS. Rev. Saúde públ., S. Paulo, 10:17-29, 1976.

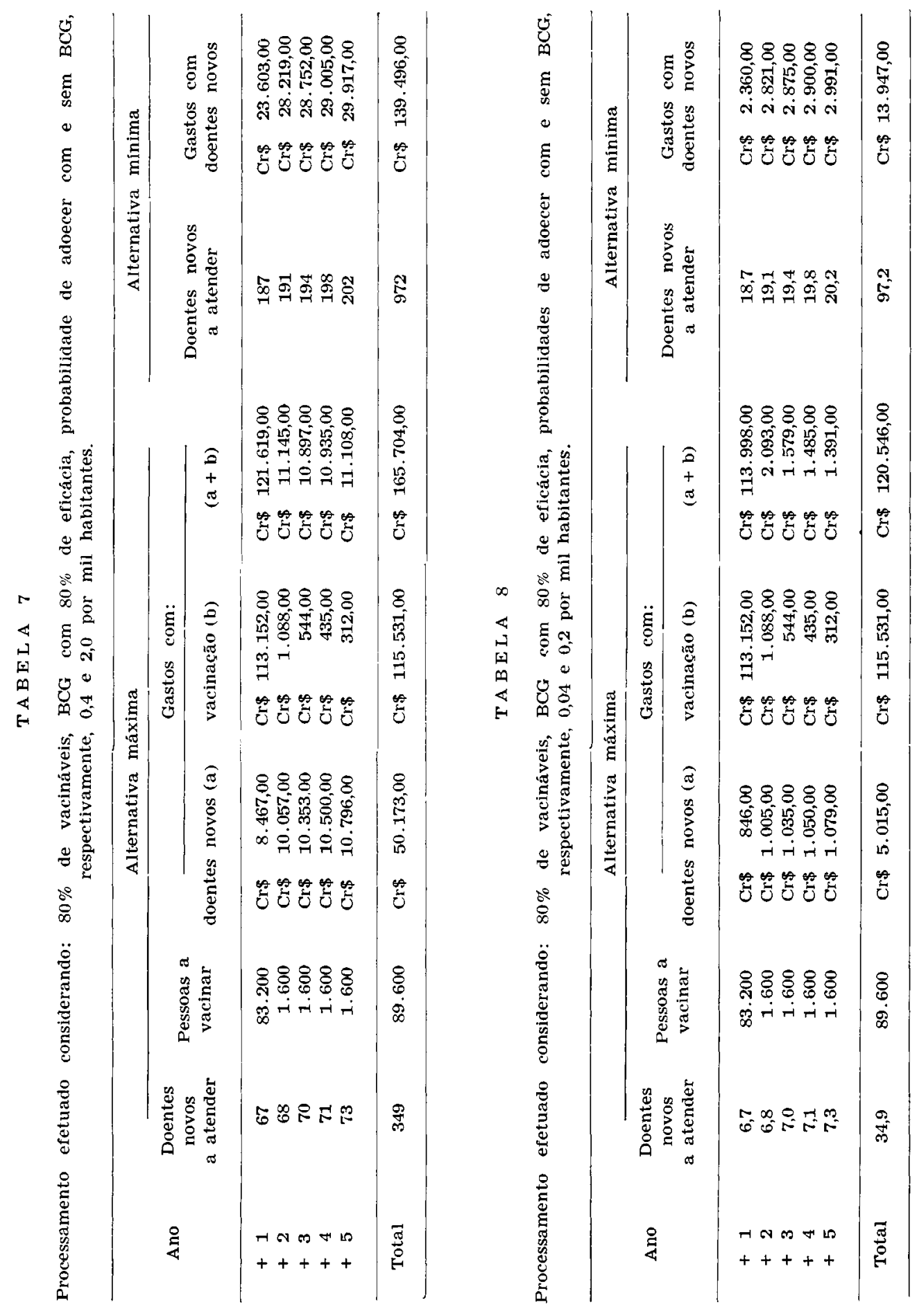


ARANTES, G. R. - Planejamento de atividades anti-tuberculose pelo método CENDES/OPS Rev. Saúde públ., S. Paulo, 10:17-29, 1976.

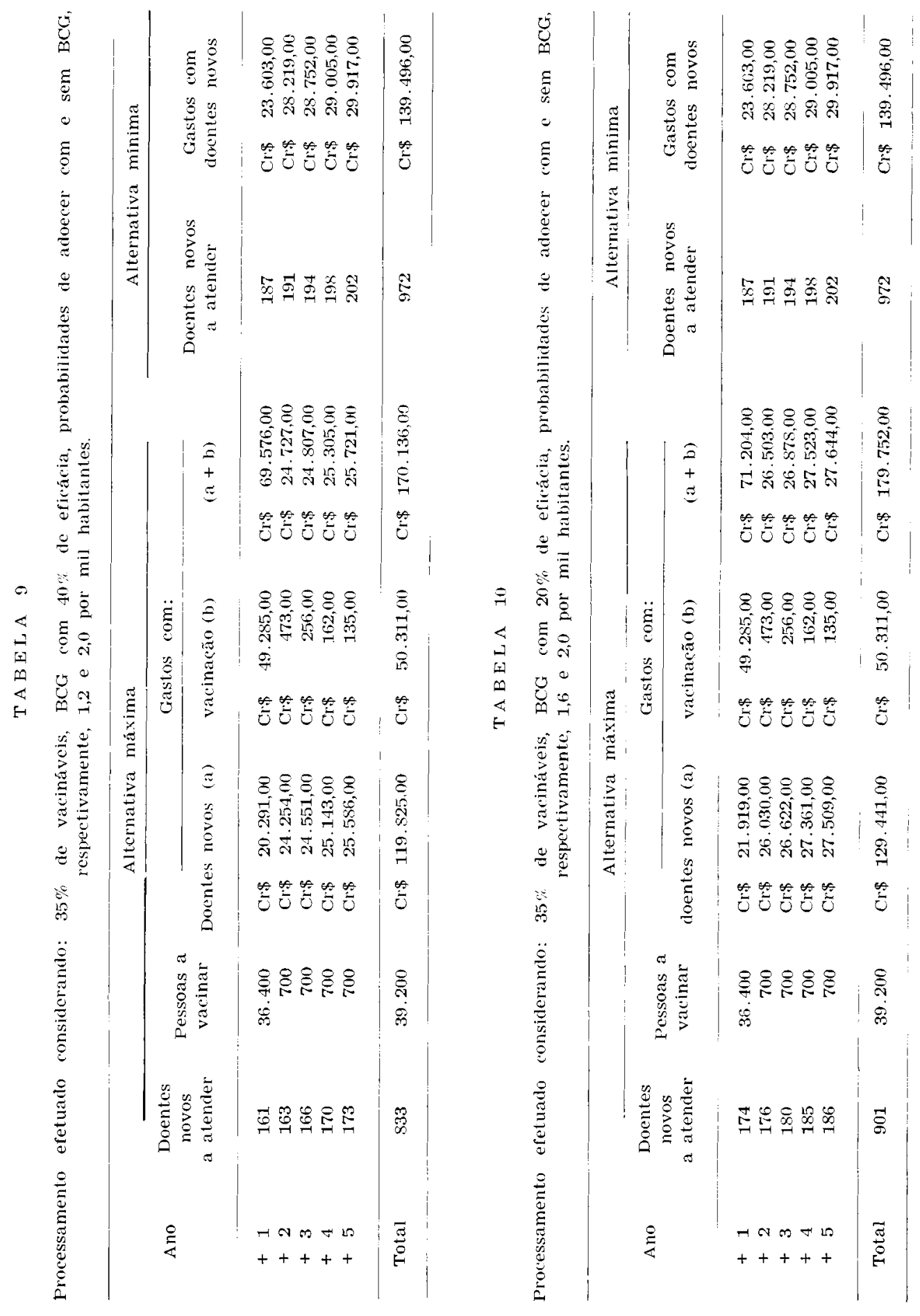


ARANTES, G. R. - Planejamento de atividades anti-tuberculose pelo método CENDES/OPS. Rev. Saúde pübl., S. Paulo, 10:17-29, 1976.

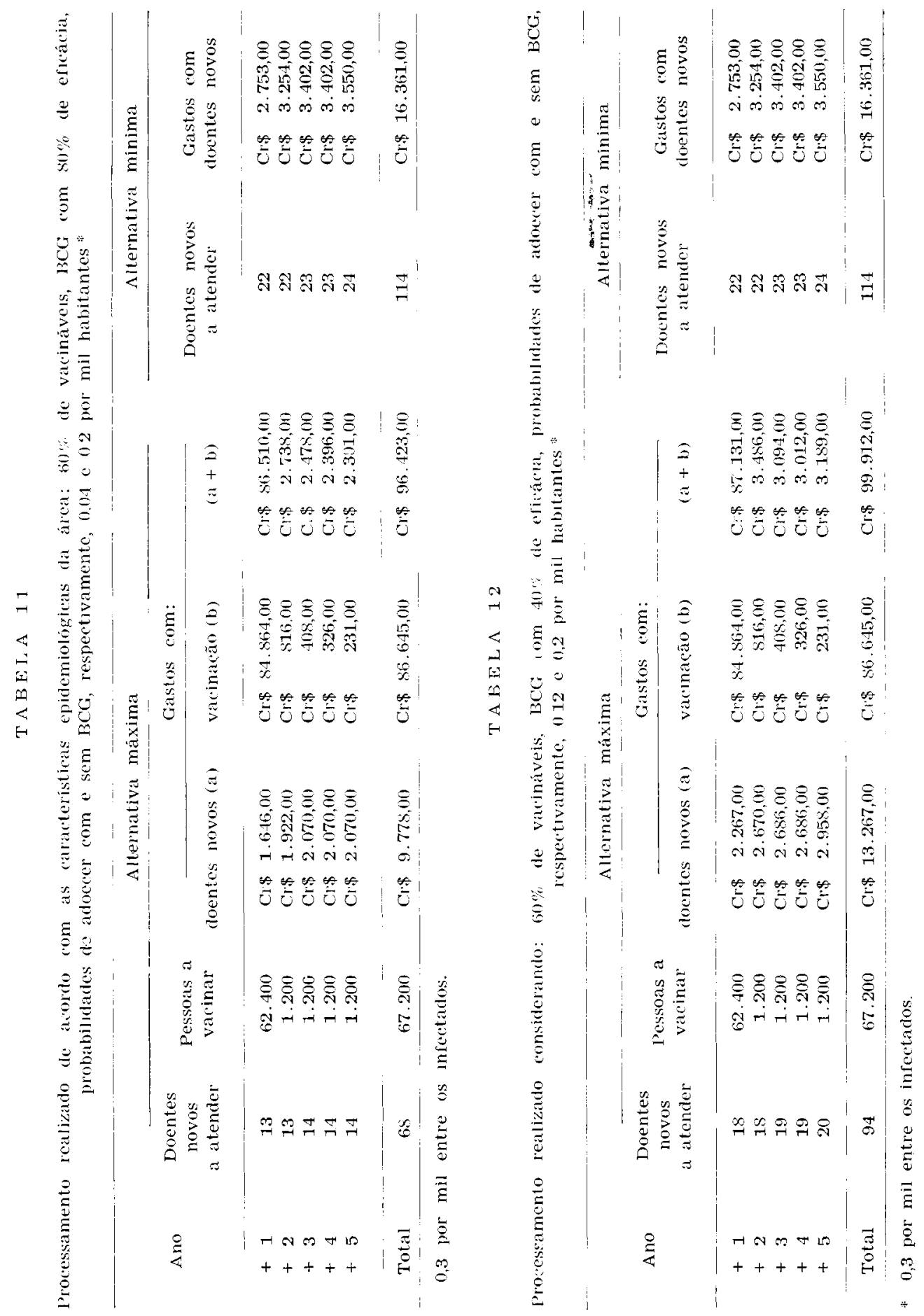


ARANTES, G. R. - Planejamento de atividades anti-tuberculose pelo método CENDES/OPS. Rev. Saúde públ., S. Paulo, 10:17-29, 1976.

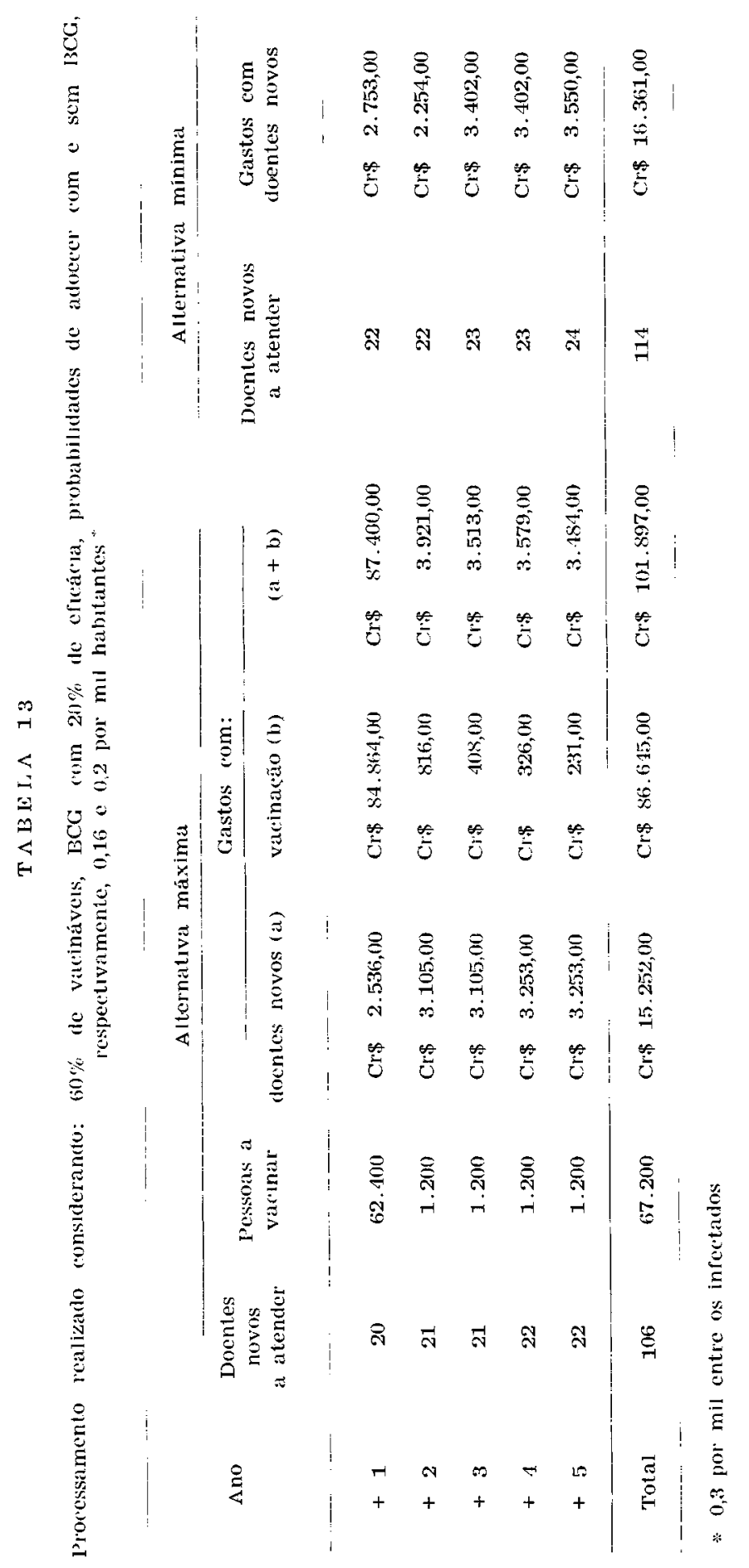


ARANTES, G. R. - Planejamento de atividades anti-tuberculose pelo método CENDES/OPS. Rev. Saúde públ., S. Paulo, 10:17-29, 1976.

nável, gastar-se-ia muito menos com vacinação, porém os gastos com doentes e o gasto total seriam superiores aos calculados segundo as normas; o inverso ocorreria se a população vacinável alcançasse a elevada proporção de $80 \%$. As alternativas máximas seriam menores que as mínimas nos 3 casos e por isso o benefício em cruzeiro por cruzeiro aplicado, maior do que um. Em nível tão baixo de prevalência de infectados (apenas 20\%), o coeficiente de incidência seria bem menor e por isso foi feito um estudo considerando a probabilidade de adoecer 10 vezes menor (Estudo n. ${ }^{\circ} 4$ ). Nessas circunstâncias as previsões orçamentárias seriam muito inferiores às 3 anteriores, porém a alternativa máxima custaria mais do que a alternativa mínima gerando um benefício-custo menor do que um.

Se a eficácia da vacina não fosse $80 \%$ a razão benefício-custo poderia cair de Cr\$ 4,56 (LS\$ 0,50) (segundo normas) para Cr\$2,34 (US\$ 0,25) ou Cr\$ 1,20 (US\$ 0,13 ) obtidos com eficácias respectivamente de $40 \%$ e $20 \%$.

Analisando-se os resultados conseguidos com os dados epidemiológicos da área (Estudo n. ${ }^{\circ} 7$ ) nota-se que os valores seriam muito menores do que aqueles obtidos aplicando-se o modelo normativo, e a razão benefício-custo menor do que um; tanto menor quanto menor a eficácia da vacina (Estudos n. ${ }^{\circ} 8$ e n. ${ }^{\circ} 9$ ).

Como a alternativa mínima risa apenas impedir que a situação se deteriore enquanto que a meta da alternativa máxima é diminuir a magnitude do problema, não será difícil optar por esta quando os seus custos forem menores.

Quando maiores, outros fatores deverão ser considerados, tais como anos-sofrimento evitados e gastos sociais com os doentes afastados do trabalho. Poderão ocorrer entretanto situaçōes em que, devida a uma incidência muito baixa da doença, um reduzido número de doentes a atender venha a custar menos do que se gastaria com um oneroso programa de vacinação, especialmente se for usada vacina de baixa eficácia. Provavelmente seria esse o caso da área programática em pauta cuja alternativa mínima para a sua realidade epidemiológica revelou-se inferior à alternativa máxima, mesmo com vacina $80 \%$ eficaz.

Todavia mais significativo é o fato de que, planejando-se segundo o modelo de normalização, as previsões orçamentárias (mínima igual a Cr\$836.976,00 - US\$ 83.692.60 - e máxima igual a Cr\$ 657.703 .00 - LS $\$ 65.770 .30$ - seriam substancialmente superiores às reais necessidades (respectivamente $\mathrm{Cr} \$ 98.166 .00$ 一 US\$9.816.60 - e Cr\$145.313,00 L'S\$14,531.30).

5 - CONCLUSOES

1 - A prevalência de infecção tuberculosa na área programática é um dado importante para a aplicação do método CENDES/OPS.

2 - A incidência de casos novos também deve ser levada em consideração.

3 - Da mesma forma é preciso conhecer pelo menos aproximadamente qual a eficácia da vacina BCG na região, para que se possa planejar adequadamente, uma vez que os gastos são inversamente proporcionais a ela.

4 - A fim de evitar uma previsão insuficiente de recursos é necessário considerar todos os doentes em tratamento e não apenas os novos doentes matriculados. 
ARANTES, G. R. - Planejamento de atividades anti-tuberculose pelo método CENDES/OPS. Rev. Saúde públ., S. Paulo, 10:17-29, 1976.

RSPU-B/296

ARANTES, G. R. - LPlanning of antituberculosis activities by the "CENDES/ PAHO" Method]. Rev. Saúde públ., S. Paulo, 10:17-29, 1976.

SUMMARY: The CENDES/PAHO method of health planning was used in the area of Araraquara, S. Paulo (Brazil). The cost of tuberculosis was determined using normative data; in order to test the influence of changes in the number of persons eligible for BCG vaccination, tuberculosis incidence and efficacy of $B C G$, simulated data were also used. Local epidemiological indices were used in order to plan antituberculosis activities. A great diversity of results showed up: costs rated by application of normative model were eight times higher than costs calculated by considering local epidemiological characteristics.

UNITERMS: Health planning. CENDES/PAHO method. Nomative model. Planning area. Epidemiological characteristics.

\section{REFERËNCIAS BIBLIOGRÁFICAS}

1. AHUMAdA, J. et al. - Problemas conceptuales y metodologicos de la programación de la salud. Washington, DC, Organización Panamericana de la Salud, 1965. (Publicación Cientifica, 111).

2. AlviM, E. F. - Análise de duas técnicas de planejamento do setor saúde Apresentado no XVIII Congresso Brasileiro de Higiene, São Paulo, 1970

3. CAMPANHA NACIONAL DE TLBERCUlose. Comissão Técnica. Prova tuberculinica em saúde pública $(2$ a recomendaçāo). Rev. Serv. nac. Tuberc., 12:219-30, 1968.

4. FaCUldade de SAÚdE PÚBlica dA USP - $V$ Curso de Planejamento do Setor Saúde. São Paulo, 1972. [Apostila].

5. HART, P. D'ARCY - Efficacy and applicability of mass $B C G$ vaccination in tuberculosis control. Brist. med. $J ., \quad 1: 587-92,1967$.
6. ORGANIZAÇAO PANAMERICANA DA SAUDE - Manual da classificaçó internacional de doenças, lesões $e$ causas de óbito. Washington, D.C.. 1969. v. 1. (Publicação Cientifica, 190).

7. OspinA, C. et al. - Planificación de la salud. Antioquia, Col. Escuela de Salud Publica de la Universidad de Antioquia, 1967.

8. SAO PAUlO (Estado). Secretaria da Saúde. Reforma administratina. Coletânea de textos legais e regulamentares. São Paulo, 1971.

9. SMITH, F. W. - Cost-effectiveness and cost benefit analyses for public health programs. Publ. Hlth. Rep., 83:899906,1968

10. SUTHERLAND, I. - Eficácia e duraçăo da proteção conferida pela vacina BCG. Rev. Serv. nac. Tuberc., 12: $177-87,1968$.

Recebido para publicaça em 03/12/1975 A provado para publucação $m$ 05/01/1976 